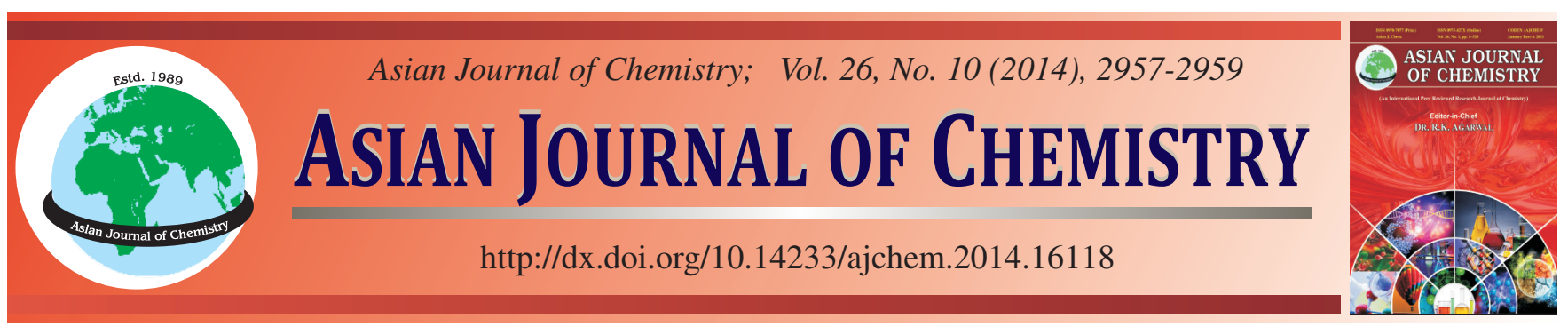

\title{
Microbial Paraffin-Removal Technology Using Paraffin-Degrading and Biosurfactant-Producing Strain
}

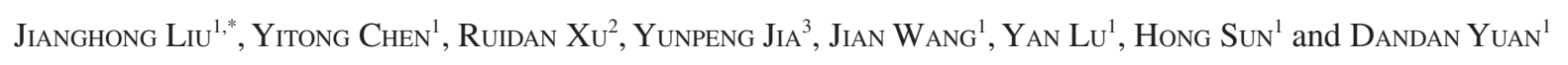

${ }^{1}$ Provincial Key Laboratory of Oil and Gas Chemical Technology, College of Chemistry and Chemical Engineering, Northeast Petroleum University, Daqing 163318, Heilongjiang, P.R. China

${ }^{2}$ School of Life sciences, Xiamen University, Xiamen 361005, Fujian Province, P.R. China

${ }^{3}$ Research Institute of Petroleum Engineering, Dagang Oilfield, Tianjin 300280, P.R. China

*Corresponding author: Tel: +86 13836967178; E-mail: 1jhread@126.com; 13836967178@126.com

The paraffin-degrading strain, isolated from waxy oil production wells in Daqing oilfield, were obtained by separation and purification experiment. The paraffin-degrading strain P1 was identified through 16S rDNA sequence analysis, which exhibited the highest similarities to Bacillus anthracis strain XFB-BN. The biosurfactant-producing strain was obtained by laboratory saved. After paraffin-degrading and biosurfactant-producing strain treatment, the paraffin degradation rate could reach to $68.2 \%$, the paraffin prevention rate could reach to $80.7 \%$, the reduction rate of crude oil viscosity was $62.4 \%$. By analyzing the data, the paraffin-degrading and biosurfactant-producing strain had better effect on microbial prevention of wax deposition.

Keywords: Paraffin-degrading, Biosurfactant-producing, Bacillus sp., Wax deposition.

\section{INTRODUCTION}

Paraffin deposition on the walls of wells and collection pipelines, which causes problems for the production and transportation of crude oil, is a common phenomenon ${ }^{1}$.

Several methods have been used to overcome the problem of paraffin deposition, including mechanical, thermal, chemical and microbial methods ${ }^{2,3}$. For mechanical treatment, which is not harm for the strata, but the stability is not strong, also is time-consuming. Thermal treatment, which is improved on paraffin removal, results in the formation damage by concentrating heavier ends of the oil and paraffin, which can be mobilized by the heat available through hot oiling, The chemical treatment, which needs less investment in equipment, is costly and highly toxic $\mathrm{c}^{4,5}$. Microbial treatment has many outstanding advantages: simple construction, low operating costs, the role of a long cycle does not affect the quality of the oil strata without any damage.

In this work, paraffin-degrading strain was obtained by separation and purification experiment from paraffinic oil production wells in Daqing Oilfield. The paraffin-degrading strain was named as P1 and biosurfactant-producing strain was named as S1. As an indicator of the degradation of paraffin, strain P1 and strain S1 were added in different proportions and then the optimum proportion was obtained. Research of mixed bacteria group has influence on the performance crude oil which can be more effective for paraffin degradation and increase the rate of melting paraffin and prevent paraffin deposition.

\section{EXPERIMENTAL}

Isolation of paraffin-degrading strain. The paraffin-degrading strain was screened from waxy oil production wells in Daqing oilfield. Experiment of identification showed that the paraffin-degrading strain P1 was identified to Bacillus sp. The 16S rDNA extraction of P1 strain was tested by Sangon Biotech (Shanghai) Co., Ltd. The 16S rDNA gene sequence obtained from the strain P1 was compared with other bacterial sequences by using blast of the National Center for Biotechnology Information (http://blast.ncbi.nlm.nih.gov/Blast.cgi). Phylogenetic trees were constructed through the neighbour-joining (NJ) algorithm available in Molecular Evolutionary Genetics Analysis (mega) Software Version 5.0. The biosurfactantproducing strain was obtained by laboratory saved.

The mixed experiment of paraffin-degrading strain P1 and biosurfactant-producing strain S1. In this experiment, as an indicator of the degradation of paraffin, the paraffin-degrading strain P1 and biosurfactant-producing strain S1 were added in different proportions. Therefore the proportions were selected as $1: 1,2: 1,3: 1,3: 2,4: 1$ and $4: 3$. Then the degradation rates of 
paraffin was measured. Marine mineral culture $(100 \mathrm{~mL})$ was sterilized by autoclaving at $121{ }^{\circ} \mathrm{C}$ for $20 \mathrm{~min}$. After sterilization, waxes $(3 \mathrm{~g})$ with the paraffin-degrading strain $(2 \mathrm{~mL})$ and the mixed paraffin-degrading strain P1 and biosurfactantproducing strain $\mathrm{S} 1$ (2 $\mathrm{mL})$, respectively, were added to the sterilized culture medium. The system was operated at the following parameters temperature $45^{\circ} \mathrm{C}, \mathrm{pH}=7.2$, salinity $5000 \mathrm{mg} / \mathrm{L}$. The incubation periods were 5, 10, 15, $20 \mathrm{~d}$. The degradation rates of paraffin were measured.

Paraffin inhibition: The method of effect evaluation was according to SY/T6300-1997. By controlling the temperature difference in crude oil and paraffin tube (crude oil was controlled at $45^{\circ} \mathrm{C}$, paraffin tube was controlled at $30^{\circ} \mathrm{C}$ ), circulating pump was operated for 14 days, which made paraffin deposit on the paraffin tube, then the paraffin tube was frozen to $25^{\circ} \mathrm{C}^{6}$. The prevention rates of paraffin were measured before microbial treatment and after microbial treatment.

Viscosity: Mixed bacteria liquid $(50 \mathrm{~mL})$ with crude oil $(50 \mathrm{~mL})$ in the conical flask was operated at the following parameters temperature $45{ }^{\circ} \mathrm{C}$. After 7 days, crude oil was dehydrated. Then the viscosity of crude oil was measured by a Rotating Viscometer (NDS-8S, China) that was set at $50{ }^{\circ} \mathrm{C}$ and $12 \mathrm{~s}^{-1}$ shear rate ${ }^{7}$.

\section{RESULTS AND DISCUSSION}

16S rDNA sequence analysis. 16S rDNA sequence analysis was shown in Fig. 1. Fig. 1. showed that through $16 \mathrm{~S}$ rDNA sequence analysis, the paraffin-degrading strain $\mathrm{P} 1$ exhibitted the highest similarities to Bacillus anthracis strain XFB-BN.

The optimum proportion of paraffin-degrading strain P1 and biosurfactant-producing strain S1. In different incubation periods, the degradation rate of paraffin after strain S1 treatment is shown in Table-1. After adding the different proportions

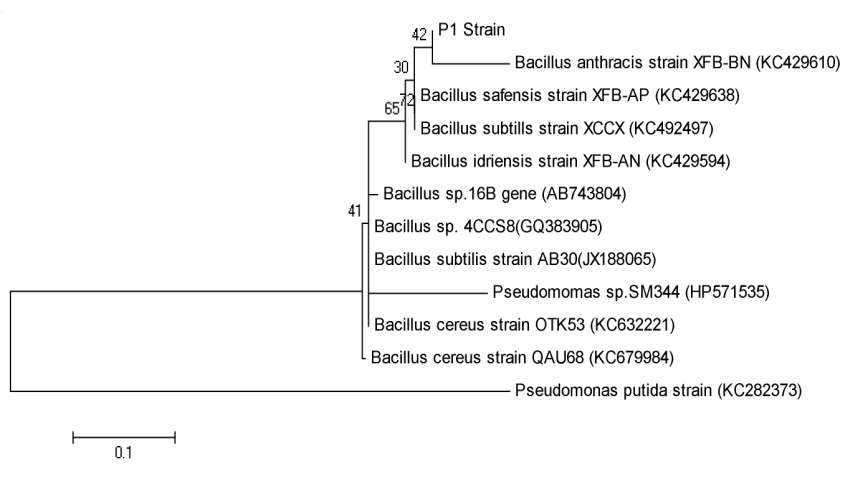

Fig. 1. Phylogenetic tree of unknown Strain P1 made in MEGA 5 software

of paraffin-degrading strain P1 and biosurfactant-producing strain $\mathrm{S} 1$, after 7 days, the degradation rate of paraffin after mixed bacteria group treatment is shown in Table-2. As shown in Table-1, strain P1 has better removal-paraffin effect, but slower removal time. As shown in Table-2, when the optimum proportion of strain $\mathrm{P} 1$ and strain $\mathrm{S} 1$ was $3: 1$, the degradation rate of paraffin could reach to $74.2 \%$, the removal time was faster. The prevention rate of paraffin after microbial treatment is shown in Table-3. Table- 3 showed that the prevention rate of paraffin could reach to $80.7 \%$ after mixed bacteria treatment, which was higher than that after strain S1 treatment. Thus it shows that mixed bacteria group has a better effect on paraffin inhibition.

Change of viscosity: The change of viscosity is shown in Table-4. Table-4 shows that the reduction rate of crude oil viscosity was $57.4 \%$ after the paraffin-degrading strain treatment. But the reduction rate of crude oil viscosity was $62.4 \%$ after the mixed bacteria group treatment. The results after the paraffin-degrading strain treatment were higher than the the results after the mixed bacteria group treatment.

TABLE-1

DEGRADATION RATE OF PARAFFIN AFTER THE PARAFFIN-DEGRADING STRAIN TREATMENT

\begin{tabular}{lccccc}
\hline Degradation time (d) & 0 & 5 & 10 & 15 & 20 \\
\hline Quality of paraffin before degradation $(\mathrm{g})$ & 3.00 & 3.00 & 3.00 & 3.00 & 3.00 \\
Quality of paraffin after degradation $(\mathrm{g})$ & 3.00 & 2.16 & 1.62 & 1.24 & 1.01 \\
Degradation rate of paraffin $(\%)$ & 0 & 28 & 46 & 58.7 & 66.3 \\
\hline
\end{tabular}

TABLE 2

DEGRADATION RATE OF PARAFFIN AFTER MIXED BACTERIA GROUP TREATMENT

\begin{tabular}{lcccccc}
\hline Proportion of strain P1 and S1 & $1: 1$ & $2: 1$ & $3: 1$ & $3: 2$ & $4: 1$ & $4: 3$ \\
\hline Degradation rate of paraffin (\%) & 54 & 61 & 74.2 & 65 & 68.2 & 63.5 \\
\hline
\end{tabular}

TABLE-3

\begin{tabular}{cccc}
\multicolumn{2}{c}{ TABLE-3 } \\
& \multicolumn{2}{c}{ PREVENTION RATE OF PARAFFIN AFTER MICROBIAL TREATMENT } \\
\cline { 2 - 3 } Strain & Weight of paraffin after 14 d $(\mathrm{g})$ & \multirow{2}{*}{ Prevention rate of paraffin $(\%)$} \\
\cline { 2 - 3 } & Before treatment & After treatment & 68.4 \\
Paraffin-degrading strain & 0.57 & 0.18 & 80.7 \\
Mixed bacteria group & 0.57 & 0.11 & 80.7 \\
\hline
\end{tabular}

TABLE-4

CRUDE OIL VISCOSITY BEFORE AND AFTER MICROBIAL TREATMENT

\begin{tabular}{|c|c|c|c|}
\hline \multirow{2}{*}{ Bacterial strain } & \multicolumn{2}{|c|}{ Crude oil viscosity (mPa-s) } & \multirow{2}{*}{ Reduction rate of viscosity (\%) } \\
\hline & Before microbial treatment & After microbial treatment & \\
\hline Paraffin-degrading strain & 48.4 & 20.6 & 57.4 \\
\hline
\end{tabular}




\section{Conclusion}

The paraffin-degrading strain, isolated from waxy oil production wells in Daqing oilfield, were obtained by separation and purification experiment. The paraffin-degrading strain P1 was identified through 16S rDNA sequence analysis, which exhibitted the highest similarities to Bacillus anthracis strain XFB-BN. The biosurfactant-producing strain was obtained by laboratory saved. After paraffin-degrading and biosurfactantproducing strain treatment, the paraffin degradation rate could reach to $68.2 \%$, the paraffin prevention rate could reach to $80.7 \%$ and the reduction rate of crude oil viscosity was $62.4 \%$. By analyzing the data, the paraffin-degrading and biosurfactant-producing strain had better effect on microbial prevention of wax deposition than the paraffin-degrading strain. So after paraffin-degrading and biosurfactant-producing strain treatment, the effect of prevention of wax deposition is higher.

\section{ACKNOWLEDGEMENTS}

This work is supported by the Science and Technology Research Project of Heilongjiang Provincial Education Department of China (12531064).

\section{REFERENCES}

1. Q. Huang, J. Wang and J. Zhang, Petroleum Sci., 6, 64 (2009).

2. S.G. Agaev, E.O. Zemlyanskii, A.N. Grebnev, S.V. Gul'tyaev and N.S. Yakovlev, Russ. J. Appl. Chem., 79, 1360 (2006).

3. A.T. Leiroz and L.F.A. Azevedo, Heat Transfer Eng., 28, 567 (2007).

4. V. Yu Loskutova, I.V. Prozorova and N.V. Yudina, Chem. Technol. Fuels Oils, 47, 358 (2011).

5. F. Lionetto, G. Coluccia, P. Dantona and A. Maffezzoli, Rheol. Acta, 46, 601 (2007).

6. J. Liu, Y. Jia and R. Xu, Asian J. Chem., 25, 5473 (2013).

7. J. Liu, Y. Chen, R. Xu and Y. Jia, Indian J. Microbiol., 53, 168 (2013). 